\title{
Excessive gestational weight gain in the first trimester is associated with risk of gestational diabetes mellitus: a prospective study from Southwest China
}

\author{
Xi Lan ${ }^{1}$, Yi-qi Zhang ${ }^{1}$, Hong-li Dong ${ }^{1}$, Ju Zhang ${ }^{2}$, Feng-ming Zhou ${ }^{1}$, Yan-hong Bao ${ }^{1}$, \\ Rong-ping Zhao ${ }^{1}$, Cong-jie Cai ${ }^{1}$, Dan Bai ${ }^{1}$, Xin-xin Pang ${ }^{1}$ and Guo Zeng ${ }^{1, *}$ \\ 'Department of Nutrition, Food Safety and Toxicology, West China School of Public Health and West China Fourth \\ Hospital, Sichuan University, No. 16, Section 3, Renmin Nan Road, 610041 Chengdu, Sichuan, People's Republic \\ of China: ${ }^{2}$ Department of Clinical Nutrition, Sichuan Provincial Hospital for Women and Children, Chengdu, Sichuan, \\ People's Republic of China
}

Submitted 15 February 2019: Final revision received 31 July 2019: Accepted 9 August 2019: First published online 20 December 2019

\begin{abstract}
Objective: To evaluate the effects of gestational weight gain (GWG) in the first trimester (GWG-F) and the rate of gestational weight gain in the second trimester (RGWG-S) on gestational diabetes mellitus (GDM), exploring the optimal GWG ranges for the avoidance of GDM in Chinese women.

Design: A population-based prospective study was conducted. Gestational weight was measured regularly in every antenatal visit and assessed by the Institute of Medicine (IOM) criteria (2009). GDM was assessed with the 75-g, 2-h oral glucose tolerance test at 24-28 weeks of gestation. Multivariable logistic regression was performed to assess the effects of GWG-F and RGWG-S on GDM, stratified by pre-pregnancy BMI. In each BMI category, the GWG values corresponding to the lowest prevalence of GDM were defined as the optimal GWG range.

Setting: Southwest China.

Participants: Pregnant women ( $n$ 1910) in 2017.

Results: After adjusting for confounders, GWG-F above IOM recommendations increased the risk of GDM (OR; 95\% CI) among underweight (2.500; 1.106, 5.655), normal-weight $(1.396 ; 1.023,1.906)$ and overweight/obese women (3.017; $1 \cdot 118,8 \cdot 138)$ compared with women within IOM recommendations. No significant difference was observed between RGWG-S and GDM $(P>0.05)$ after adjusting for GWG-F based on the previous model. The optimal GWG-F ranges for the avoidance of GDM were $0 \cdot 8-1 \cdot 2,0 \cdot 8-1.2$ and $0.35-0.70 \mathrm{~kg}$ for underweight, normal-weight and overweight/obese women, respectively.

Conclusions: Excessive GWG in the first trimester, rather than the second trimester, is associated with increased risk of GDM regardless of pre-pregnancy BMI. Obstetricians should provide more pre-emptive guidance in achieving adequate GWG-F.
\end{abstract}

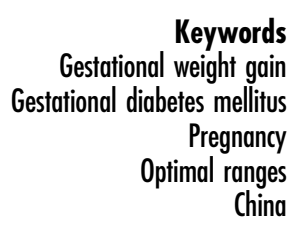

Gestational diabetes mellitus (GDM) is defined as any degree of glucose intolerance with onset or first recognition during pregnancy ${ }^{(1)}$. GDM is associated with an increased risk of perinatal complications ${ }^{(2-4)}$ and in the long term, women with $\mathrm{GDM}^{(5)}$ and their offspring are at elevated risk of developing obesity and type 2 diabetes mellitus ${ }^{(6)}$. Given the rise in GDM incidence over the past decades in China $^{(7,8)}$, preventing GDM has become a public health priority.
Previous studies have reported that excessive gestational weight gain (GWG) may result in maternal fat deposition, which may decrease insulin sensitivity. It is biologically plausible that GWG is positively associated with $\mathrm{GDM}^{(9)}$. Previously, most related studies have focused on the association between total GWG and GDM, but the result could not be factually reflected due to clinical intervention after GDM diagnosis, and even reverse causation might be obtained ${ }^{(10)}$. Recently, studies have examined 
the association between GWG prior to the GDM screening test and the risk of GDM, but the conclusions are not consistent. Several case-control studies have shown that GWG prior to the GDM screening test, especially gestational weight gain in the first trimester (GWG-F), was associated with the risk of GDM, while an association between gestational weight gain in the second trimester (GWG-S) and GDM was not observed ${ }^{(11-13)}$. One cohort study found that greater early GWG was associated with increased risk of GDM and elevated weight gain in mid-pregnancy increased the risk of GDM only among pregnant women with greater weight gain in the first trimester $^{(14)}$. Several studies have also examined the association between GWG prior to the GDM screening test and the risk of GDM in women with different prepregnancy BMI, but the reported data were controversial. One study found that the rate of gestational weight gain in the first trimester (RGWG-F) was significantly associated with GDM in the normal-weight group, but not in the underweight or obese group ${ }^{(15)}$. Another study showed that GWG is a significant risk factor for GDM in overweight or obese women but not in women who were underweight or had a normal pre-pregnancy $\mathrm{BMI}^{(16)}$. At present, it remains unclear whether trimester-specific GWG prior to glucose screening is associated with an elevated risk of GDM in women with different pre-pregnancy BMI.

GWG guidelines were published by the US Institute of Medicine (IOM) in $1990^{(17)}$ and revised in 2009 $9^{(9)}$. However, they may be of limited use in Asian populations because they are largely based upon studies of Caucasian women. Furthermore, the WHO international BMI cut-off points defining pre-pregnancy BMI categories in the IOM guidelines are not directly applicable to Asians, especially the Chinese. Most importantly, the IOM does not take the pregnancy outcome of GDM into consideration. A study to define optimal GWG ranges for the avoidance of GDM was needed for Chinese women.

\section{Methods}

\section{Study sample and design}

Participants for the present analysis were derived from a population-based prospective study conducted in Sichuan Provincial Hospital for Women and Children, Southwest China, which was designed to investigate the effects of GWG and serum lipids on GDM. All participants provided written informed consent when recruited to the cohort.

Women who had their first prenatal visit during the first trimester were invited to join this cohort in 2017. Initially, 2211 pregnant women with weight measurement at $12 \pm 2$ weeks of gestation were recruited for the study. Of all these participants, 178 were excluded for induced labour ( $n$ 143), multiple births ( $n$ 32) and diabetes diagnosed before pregnancy ( $n 3)$. Participants who had missing data on maternal height or pre-pregnancy weight $(n 2)$ or who did not have information about the result of the oral glucose tolerance test (OGTT; $n$ 121) were also excluded. Therefore, 1910 pregnant women ( $86.4 \%$ of the eligible sample) were eventually obtained for the statistical analyses.

\section{Data collection and anthropometric measurements}

All participants completed a questionnaire about maternal demographics, weeks of gestation, parity, family history of diabetes, pre-pregnancy drinking and smoking habits, physical activity, psychological state, sleep quality during early pregnancy and dietary intake in the last $3 \mathrm{~d}$. The questionnaire was given by trained staff in face-to-face interviews when participants enrolled. Physical activity, sleep quality, dietary intake and weeks of gestation in the second trimester were also recorded when participants had their prenatal care visit at 24-28weeks of gestation. The 75-g OGTT was conducted at 24-28 weeks of gestation. The OGTT result was extracted from the electronic medical record system maintained by the hospital.

Maternal pre-pregnancy weight was based on selfreporting. High correlations were found between self-reported and measured pre-pregnancy weight ${ }^{(18)}$. Height in metres was measured on standardized height-measuring stations at the first prenatal visit. Weight in kilograms was measured on standardized digital weighing scales by trained staff at the first prenatal visit and at 24-28 weeks of gestation.

\section{Gestational weight gain}

GWG-F was calculated by subtracting pre-pregnancy weight from maternal weight at $12 \pm 2$ weeks of gestation. The rate of gestational weight gain in the second trimester (RGWG-S) was calculated as follows: RGWG-S = (measured weight at 24-28 weeks - measured weight at $12 \pm 2$ weeks)/weeks between the two measurements. GWG-F and RGWG-S were classified by the cut-offs of the IOM recommendations ${ }^{(9)}$ into three groups: below, within and above the IOM recommendations. In recent years, most hospitals have used the BMI cut-off points in China when applying IOM GWG recommendations to Chinese women. Therefore, the BMI cut-off points in China were adopted to determine pre-pregnancy weight status when applying IOM GWG recommendations to classify participants into the three groups of below, within and above the IOM recommendations. For instance, in terms of GWG-F, the IOM recommends the same range regardless of pre-pregnancy BMI. Therefore, our study classified pregnant women as below when GWG-F was less than $0.5 \mathrm{~kg}$, within when GWG-F was between 0.5 and $2 \mathrm{~kg}$, and above when GWG-F was more than $2 \mathrm{~kg}$.

\section{Diagnostic criteria of gestational diabetes mellitus}

At 24-28 weeks of gestation, the 75-g OGTT was conducted. GDM was diagnosed according to the standards 
issued by the Ministry of Health of China in 2011, which were based on the International Association of Diabetic Pregnancy Study Group guidelines ${ }^{(19)}$. Briefly, GDM was defined as having one or more abnormal values from a 75-g, 2-h OGTT between 24 and 28 weeks of gestation, with cut-off points of $5.1 \mathrm{mmol} / 1(92 \mathrm{mg} / \mathrm{dl})$ for fasting, $10.0 \mathrm{mmol} / \mathrm{l}(180 \mathrm{mg} / \mathrm{dl})$ for 1 -h post-load and $8.5 \mathrm{mmol} / \mathrm{l}$ $(153 \mathrm{mg} / \mathrm{dl})$ for 2 -h post-load glucose. In China, women diagnosed with GDM receive both diet and physical activity guidelines at the hospital, which was the basis of selecting women for the groups with GWG in the first and second trimesters prior to the diagnosis of GDM.

\section{Additional information}

On the basis of self-reported pre-pregnancy weight and height measured at the first prenatal visit, pre-pregnancy BMI was calculated as self-reported pre-pregnancy weight in kilograms divided by squared height in metres and was categorized as underweight $\left(\mathrm{BMI}<18.5 \mathrm{~kg} / \mathrm{m}^{2}\right)$, normal-weight $\quad\left(18.5 \leq \mathrm{BMI}<24.0 \mathrm{~kg} / \mathrm{m}^{2}\right)$, overweight $\left(24.0 \leq \mathrm{BMI}<28.0 \mathrm{~kg} / \mathrm{m}^{2}\right)$ or obese $\left(\mathrm{BMI} \geq 28.0 \mathrm{~kg} / \mathrm{m}^{2}\right)$ according to the Chinese criteria ${ }^{(20)}$. As a result of the descriptive similarities and small numbers, overweight and obese women were combined in the analysis.

Gestational age was calculated based on the earliest ultrasonogram obtained. Educational level was classified as $\leq 12,13-15$ and $\geq 16$ years according to the number of completed schooling years. Drinking before 6 months of pregnancy was considered positive when the woman had drunk alcohol before 6 months of pregnancy. Exposure to a second-hand smoking environment frequently before 6 months of pregnancy was defined as staying in a secondhand smoking environment more than three times per week before 6 months of pregnancy. Regular exercise before pregnancy referred to exercise more than three times per week before pregnancy.

\section{Statistical analysis}

Continuous variables are presented as means and standard deviations. Categorical variables are expressed as frequencies and percentages. The $\chi^{2}$ test was performed to analyse the relationship between variables. Participants were categorized into three groups (below, within and above the IOM recommendations) on account of the GWG-F and RGWG-S status. Multivariable logistic regression was used to assess the effects of GWG-F and RGWG-S on GDM stratified by pre-pregnancy BMI, adjusting for the following confounding variables: maternal age $(<25,25-29$ and $30-34$ years, $\geq 35$ years as reference), educational level ( $\leq 12$ and $13-15$ years, $\geq 16$ years as reference), parity (primiparous or multiparous), employment (yes or no), family history of diabetes (yes or no), family history of hypertension (yes or no), family history of thyroid diseases (yes or no), average personal income per month $(<3000$, 3000-4999 and 5000-9999 Yuan, $\geq 10000$ Yuan as reference), regular exercise before pregnancy (yes or no), frequent exposure to a second-hand smoking environment (yes or no) and drinking before 6 months of pregnancy (yes or no). Studies have found that these variables, including regular exercise before pregnancy, family history of thyroid diseases, average personal income per month, frequent exposure to a second-hand smoking environment and drinking before pregnancy, were related to $\mathrm{GDM}^{(21-25)}$, so our study adjusted for them in the final models. Women whose GWG-F and RGWG-S were within the IOM recommendations were used as the reference group. All confidence intervals were estimated at the $95 \%$ level and $P<0.05$ was considered statistically significant.

To explore the optimal GWG ranges for the avoidance of GDM for Chinese women, the prevalence of GDM was calculated when GWG increased by a certain unit. The present study defined the optimal GWG ranges stratified by China-specific BMI categories ${ }^{(20)}$ as the corresponding GWG ranges when the prevalence of GDM was lowest. As a result of the descriptive similarities and small numbers, overweight and obese women were also combined in this analysis. All data were analysed using the statistical software package IBM SPSS Statistics version 21.0.

\section{Results}

Among the 1910 participants in the study, 620 (32.5\%) were diagnosed with GDM. The mean maternal age was 28.7 (SD 4.0) years and most of the women were primiparous (75.9\%). Compared with women in the non-GDM group, women with GDM were more likely to be older, to have a higher pre-pregnancy BMI and to have a family history of diabetes or hypertension $(P<0.05)$. A higher proportion of unemployment was observed among women with GDM $(P<0.05)$. The mean GWG-F and RGWG-S were $0.7(\mathrm{SD} 2.5) \mathrm{kg}$ and $0.5(\mathrm{SD} 0.2) \mathrm{kg} /$ week, respectively. GWG-F was significantly higher in the GDM group, but RGWG-S was lower than the non-GDM group (Table 1).

Table 2 shows the results of a multivariable logistic regression analysis for the effects of GWG-F and RGWG-S on GDM stratified by pre-pregnancy BMI. After adjusting for maternal age, educational level, parity, employment, family history of diabetes, family history of hypertension, family history of thyroid diseases, average personal income per month, regular exercise before pregnancy, frequent exposure to a second-hand smoking environment and drinking before 6 months of pregnancy, our study found that GWG-F above the IOM recommendations increased the risk of GDM among underweight $(\mathrm{OR}=2.500 ; 95 \%$ CI $1 \cdot 106$, 5.655), normal-weight (OR $=1.396 ; 95 \%$ CI $1.023,1.906)$ and overweight/obese women (OR $=3 \cdot 017 ; 95 \%$ CI $1 \cdot 118$, $8 \cdot 138)$ compared with women within IOM recommendations. No significant difference was observed between RGWG-S and GDM $(P>0.05)$ in different pre-pregnancy 
Table 1 Characteristics of the study sample of pregnant women $(n 1910)$ from Southwest China, 2017

\begin{tabular}{|c|c|c|c|c|c|c|c|}
\hline \multirow[b]{2}{*}{ Characteristic $^{*}$} & \multicolumn{2}{|c|}{ Total $(n 1910)$} & \multicolumn{2}{|c|}{ Non-GDM ( $n 1290)$} & \multicolumn{2}{|c|}{ GDM $(n 620)$} & \multirow[b]{2}{*}{$P+$} \\
\hline & Mean or $n$ & SD or $\%$ & Mean or $n$ & SD or $\%$ & Mean or $n$ & SD or $\%$ & \\
\hline Gestational age at enrolment (weeks) & $11 \cdot 1$ & 1.8 & $11 \cdot 1$ & 1.8 & $11 \cdot 1$ & 1.8 & 0.837 \\
\hline $\begin{array}{l}\text { Gestational age at weight measured in the second } \\
\text { trimester (weeks) }\end{array}$ & $25 \cdot 5$ & 1.8 & $25 \cdot 6$ & 1.9 & $25 \cdot 2$ & 1.8 & $<0.001$ \\
\hline Weight at enrolment $(\mathrm{kg})$ & $52 \cdot 9$ & 7.9 & $52 \cdot 1$ & $7 \cdot 6$ & 54.5 & $8 \cdot 3$ & $<0.001$ \\
\hline Maternal age (years) & 28.7 & 4.0 & $28 \cdot 3$ & $3 \cdot 8$ & $29 \cdot 6$ & 4.2 & $<0.001$ \\
\hline$<25$ years & 160 & 8.4 & 121 & $9 \cdot 4$ & 39 & $6 \cdot 3$ & $<0.001$ \\
\hline $25-29$ years & 1035 & $54 \cdot 2$ & 747 & $57 \cdot 9$ & 288 & $46 \cdot 5$ & \\
\hline $30-35$ years & 529 & $27 \cdot 7$ & 324 & $25 \cdot 1$ & 205 & 33.1 & \\
\hline$\geq 35$ years & 186 & 9.7 & 98 & $7 \cdot 6$ & 88 & $14 \cdot 2$ & \\
\hline Pre-pregnancy BMI $\left(\mathrm{kg} / \mathrm{m}^{2}\right)$ & 20.9 & $2 \cdot 7$ & 20.5 & $2 \cdot 6$ & 21.5 & 2.8 & $<0.001$ \\
\hline Underweight $\left(<18.5 \mathrm{~kg} / \mathrm{m}^{2}\right)$ & 336 & $17 \cdot 6$ & 276 & $21 \cdot 4$ & 60 & 9.7 & $<0.001$ \\
\hline Normal $\left(18.5-23.9 \mathrm{~kg} / \mathrm{m}^{2}\right)$ & 1348 & $70 \cdot 6$ & 887 & $68 \cdot 8$ & 461 & $74 \cdot 4$ & \\
\hline Overweight/obese $\left(\geq 24.0 \mathrm{~kg} / \mathrm{m}^{2}\right)$ & 226 & $11 \cdot 8$ & 127 & $9 \cdot 8$ & 99 & $16 \cdot 0$ & \\
\hline GWG-F (kg) & 0.7 & 2.5 & 0.6 & 2.5 & 0.9 & 2.5 & $<0.05$ \\
\hline Underweight & 0.9 & 1.8 & 0.9 & 1.9 & 1.0 & 1.7 & 0.708 \\
\hline Normal & 0.6 & $2 \cdot 5$ & 0.5 & 2.5 & 0.9 & 2.4 & $<0.05$ \\
\hline Overweight/obese & 0.6 & 3.4 & 0.5 & 3.5 & 0.6 & $3 \cdot 3$ & 0.834 \\
\hline RGWG-S (kg/week) & 0.5 & 0.2 & 0.5 & 0.2 & 0.4 & 0.2 & $<0.001$ \\
\hline Underweight & 0.6 & 0.2 & 0.6 & 0.2 & 0.5 & 0.2 & 0.082 \\
\hline Normal & 0.5 & 0.2 & 0.5 & 0.2 & 0.4 & 0.2 & $<0.001$ \\
\hline Overweight/obese & 0.4 & 0.2 & 0.4 & 0.2 & 0.3 & 0.3 & 0.135 \\
\hline Ethnology (Han Chinese) & 1867 & $97 \cdot 8$ & 1261 & $97 \cdot 8$ & 606 & $97 \cdot 7$ & 0.905 \\
\hline \multicolumn{8}{|l|}{ Parity } \\
\hline Primiparous & 1446 & $75 \cdot 9$ & 1012 & $78 \cdot 7$ & 434 & $70 \cdot .0$ & $<0.001$ \\
\hline Multiparous & 460 & $24 \cdot 1$ & 274 & $21 \cdot 3$ & 186 & 30.0 & \\
\hline \multicolumn{8}{|l|}{ Education (schooling years) } \\
\hline$\leq 12$ & 431 & $22 \cdot 8$ & 292 & $22 \cdot 9$ & 139 & $22 \cdot 5$ & 0.905 \\
\hline$\overline{13}-15$ & 631 & 33.4 & 428 & 33.6 & 203 & 32.9 & \\
\hline$\geq 16$ & 829 & 43.8 & 554 & 43.5 & 275 & $44 \cdot 6$ & \\
\hline \multicolumn{8}{|l|}{ Average personal income per month (Yuan) } \\
\hline$<3000$ & 107 & $5 \cdot 6$ & 72 & $5 \cdot 6$ & 35 & $5 \cdot 7$ & 0.188 \\
\hline $3000-4999$ & 582 & $30 \cdot 6$ & 408 & 31.8 & 174 & $28 \cdot 2$ & \\
\hline 5000-9999 & 819 & 43.1 & 555 & $43 \cdot 3$ & 264 & 42.9 & \\
\hline$\geq 10000$ & 391 & $20 \cdot 6$ & 248 & $19 \cdot 3$ & 143 & $23 \cdot 2$ & \\
\hline Employment & 1267 & $66 \cdot 6$ & 886 & $69 \cdot 1$ & 381 & 61.5 & 0.001 \\
\hline Family history of diabetes & 206 & $10 \cdot 8$ & 126 & $9 \cdot 8$ & 80 & 12.9 & 0.039 \\
\hline Family history of hypertension & 505 & $26 \cdot 4$ & 321 & 24.9 & 184 & 29.7 & 0.026 \\
\hline Family history of thyroid diseases & 94 & 4.9 & 67 & 5.2 & 27 & 4.4 & 0.427 \\
\hline Regular exercise before pregnancy & 496 & $26 \cdot 0$ & 330 & $25 \cdot \overline{6}$ & 166 & $26 \cdot 8$ & 0.577 \\
\hline Drinking before 6 months of pregnancy & 98 & $5 \cdot 1$ & 63 & 4.9 & 35 & $5 \cdot 6$ & 0.489 \\
\hline $\begin{array}{l}\text { Frequent exposure to a second-hand smoking environment } \\
\text { frequently before } 6 \text { months of pregnancy }\end{array}$ & 500 & $26 \cdot 2$ & 353 & $27 \cdot 4$ & 147 & 23.7 & 0.087 \\
\hline
\end{tabular}

GDM, gestational diabetes mellitus; GWG-F, gestational weight gain in the first trimester; RGWG-S, rate of gestational weight gain in the second trimester.

*The data are presented as mean and SD for continuous variables or as $n$ and $\%$ for categorical variables.

†Tests for differences between GDM and non-GDM were performed using the Wilcoxon two-sample test for non-normally distributed continuous variables or the $\chi^{2}$ test for categorical variables; $P<0.05$ indicates significance.

BMI groups after adjusting for GWG-F based on the previous model.

The prevalence of GDM in different pre-pregnancy BMI groups when GWG-F increased by a certain level was calculated. To ensure a quantitative balance between groups, the interval unit for underweight and normal-weight groups was determined to be $0.4 \mathrm{~kg}$. The interval unit for the overweight/obese group was $0.35 \mathrm{~kg}$ because the total GWG-F was less than in the other two groups. The optimal GWG-F ranges stratified by China-specific BMI categories were set as the corresponding ranges when the prevalence of GDM was lowest. The lowest prevalence of GDM occurred in women who gained $0.8-1.2,0.8-1.2$ and $0.35-0.7 \mathrm{~kg}$ in the first trimester among underweight, normal-weight and overweight/obese women, respectively (Table 3). The optimal GWG-F for the avoidance of GDM was $0.8-1.2 \mathrm{~kg}$ in both underweight and normal-weight women, while overweight/obese women had the optimal GWG-F range of $0.35-0.7 \mathrm{~kg}$. The optimal ranges along with corresponding IOM recommendations for different pre-pregnancy BMI groups are given in Table 4. The optimal GWG-F ranges in our study were within IOM recommendations, while the upper limit for each pre-pregnancy BMI group was lower than that in the IOM recommendations.

\section{Discussion}

The present study is the first to investigate the optimal GWG ranges for the avoidance of GDM stratified by 
Table 2 The effects of gestational weight gain in the first trimester (GWG-F) and rate of gestational weight gain in the second trimester (RGWG-S) on gestational diabetes mellitus (GDM), stratified by pre-pregnancy $\mathrm{BMI}$, in the study sample of pregnant women ( $n 1910)$ from Southwest China, 2017

\begin{tabular}{|c|c|c|c|c|c|}
\hline & \multicolumn{2}{|c|}{ GDM } & \multirow[b]{2}{*}{ Adjusted OR } & \multirow[b]{2}{*}{$95 \% \mathrm{Cl}$} & \multirow[b]{2}{*}{$P$} \\
\hline & $n$ & $\%$ & & & \\
\hline \multicolumn{6}{|c|}{ GWG-F* } \\
\hline \multicolumn{6}{|c|}{ Underweight } \\
\hline Within & 22 & $16 \cdot 9$ & 1.000 & Ref. & - \\
\hline Below & 21 & $15 \cdot 7$ & 1.011 & $0.488,2.095$ & 0.977 \\
\hline Above & 17 & 23.6 & 2.500 & $1 \cdot 106,5 \cdot 655$ & $<0.05$ \\
\hline \multicolumn{6}{|l|}{ Normal } \\
\hline Within & 122 & $34 \cdot 2$ & 1.000 & Ref. & - \\
\hline Below & 198 & 30.4 & 0.853 & $0.646,1.126$ & 0.262 \\
\hline Above & 141 & 41.7 & 1.396 & $1.023,1.906$ & $<0.05$ \\
\hline \multicolumn{6}{|c|}{ Overweight/obese } \\
\hline Within & 21 & $38 \cdot 2$ & 1.000 & Ref. & - \\
\hline Below & 43 & 43.0 & $2 \cdot 060$ & $0.818,5.188$ & 0.125 \\
\hline Above & 35 & $49 \cdot 3$ & 3.017 & $1 \cdot 118,8 \cdot 138$ & $<0.05$ \\
\hline \multicolumn{6}{|c|}{ RGWG-S† } \\
\hline \multicolumn{6}{|c|}{ Underweight } \\
\hline Within & 16 & $16 \cdot 2$ & 1.000 & Ref. & - \\
\hline Below & 24 & 24.7 & 1.724 & $0.817,3.637$ & 0.153 \\
\hline Above & 17 & $12 \cdot 9$ & 0.841 & $0.386,1.835$ & 0.664 \\
\hline \multicolumn{6}{|l|}{ Normal } \\
\hline Within & 184 & $37 \cdot 2$ & 1.000 & Ref. & - \\
\hline Below & 121 & $41 \cdot 3$ & $1 \cdot 109$ & $0.790,1.557$ & 0.552 \\
\hline Above & 144 & $27 \cdot 2$ & 0.744 & $0.550,1.006$ & 0.055 \\
\hline \multicolumn{6}{|c|}{ Overweight/obese } \\
\hline Within & 23 & $46 \cdot 0$ & 1.000 & Ref. & - \\
\hline Below & 25 & $54 \cdot 3$ & 1.219 & $0.501,2.965$ & 0.662 \\
\hline Above & 49 & $39 \cdot 2$ & 0.757 & $0.365,1.569$ & 0.453 \\
\hline
\end{tabular}

Ref., reference category.

*Adjusted for maternal age, educational level, parity, employment, family history of diabetes, family history of hypertension, family history of thyroid diseases, average personal income per month, regular exercise before pregnancy, frequent exposure to a second-hand smoking environment before 6 months of pregnancy, and drinking before 6 months of pregnancy.

†Adjusted for GWG-F based on the previous model.

China-specific BMI categories. Our study found that excessive GWG-F in women with different pre-pregnancy BMI, rather than RGWG-S, was associated with an increased risk of GDM. The optimal GWG-F ranges for the avoidance of GDM were $0.8-1.2 \mathrm{~kg}$ in both underweight and normalweight women, while overweight/obese women had the optimal GWG-F range of $0.35-0.7 \mathrm{~kg}$. The IOM recommends the same range of weight gain in the first trimester regardless of pre-pregnancy BMI. This is the greatest difference between our study and the IOM recommendations. The optimal GWG-F ranges in our study were within IOM recommendations, while the upper limit for each pre-pregnancy BMI group was lower than that in the IOM recommendations.

Our finding that excessive GWG-F increased the risk of GDM in women with different pre-pregnancy BMI is consistent with several studies ${ }^{(12,26-28)}$. In a prospective cohort study, Dong et al. found that excessive weight gain in the first trimester was significantly related to a higher risk of developing GDM, with an OR of 2.655 (95\% CI $1.265,5.571)^{(26)}$. Hedderson et al. found that greater rate of GWG, especially in the first trimester, increased the risk of GDM after adjusting for potential confounders ${ }^{(12)}$. Zhong et al. demonstrated that greater early pregnancy weight gain was associated with increased risk of $\mathrm{GDM}^{(28)}$. Carreno et al. reported that only normal-weight women with excessive early GWG had a higher increase in the risk of developing GDM. Similar results were not found in underweight and overweight or obese women ${ }^{(29)}$. However, Saldana et al. have suggested that obese women may already be at a high insulin resistance level or have $\beta$ cells closer to a point of exhaustion at the start of their pregnancy, thus limiting the potential effect on glucose intolerance of additional weight gain during pregnancy ${ }^{(30)}$. Gibson et al. found that GWG before GDM diagnosis was a significant risk factor for GDM in overweight or obese women but not in women who were underweight or had a normal BMI before pregnancy ${ }^{(16)}$. Excessive GWG-F may result in an increase in maternal fat mass, which decreases insulin sensitivity and increases insulin resistance, leading to the development of GDM. However, Herring et al. found that higher weight gain prior to GDM diagnosis conferred an increased risk of impaired glucose tolerance but not GDM when they examined the association between GWG before GDM diagnosis and maternal hyperglycaemia ${ }^{(31)}$.

Because the weight gain composition during gestation (fat and fetal mass proportions) and the maternal metabolism changes vary by trimester ${ }^{(12,32)}$, it seems that the timing of excessive weight gain during the different trimesters of pregnancy has different effects on GDM. In the current study, we found that RGWG-S was not associated with GDM in any pre-pregnancy BMI group, similar to a recent study. Those authors examined the relationship between weight gain trajectories in the first and second trimesters of pregnancy and GDM among 806 women with GDM and 4819 randomly sampled women without GDM in a case-cohort study, concluding that the gain trajectory of the second trimester was not associated with GDM $(\mathrm{OR}=1 \cdot 1 ; 95 \% \mathrm{CI} 0.9,1.3)^{(33)}$. Morisset et al. found that the rate of GWG until GDM diagnosis was a risk factor for GDM. However, only first-trimester weight gain was an independent predictor of GDM. In fact, second-trimester weight gain was not associated with GDM risk ${ }^{(11)}$. Liu et al. demonstrated that elevated weight gain in mid-pregnancy increased the risk of GDM only among pregnant women with greater weight gain in the first trimester ${ }^{(14)}$. However, Hantoushzadeh et al. explored the effects of changes in indicators of adiposity and found that gains in gestational weight increased the risk of $\mathrm{GDM}^{(27)}$, although they did not consider the effect of the interaction of weight gain between both periods and risk of GDM. As weight gain in the first trimester is disproportionately composed of adipose tissue compared with that in later pregnancy $^{(32)}$, it can be hypothesized that the increasing fat mass in the first trimester may play a relatively important role in glucose intolerance.

The present study has also analysed RGWG-F and GWG-S, producing the same results as GWG-F and 
Table 3 The prevalence of certain levels of gestational weight gain in the first trimester (GWG-F) according to pre-pregnancy BMI among the study sample of pregnant women (n 1910) from Southwest China, 2017

\begin{tabular}{|c|c|c|c|c|c|c|c|c|c|c|c|}
\hline \multicolumn{4}{|c|}{ Underweight } & \multicolumn{4}{|c|}{ Normal weight } & \multicolumn{4}{|c|}{ Overweight/obese } \\
\hline \multirow[b]{2}{*}{ GWG-F (kg) } & \multirow[b]{2}{*}{$n$} & \multicolumn{2}{|c|}{ GDM } & \multirow[b]{2}{*}{ GWG-F (kg) } & \multirow[b]{2}{*}{$n$} & \multicolumn{2}{|c|}{ GDM } & \multirow[b]{2}{*}{ GWG-F (kg) } & \multirow[b]{2}{*}{$n$} & \multicolumn{2}{|c|}{ GDM } \\
\hline & & $n$ & $\%$ & & & $n$ & $\%$ & & & $n$ & $\%$ \\
\hline$<0$ & 109 & 16 & $14 \cdot 7$ & $<0$ & 539 & 160 & 29.7 & $<0$ & 88 & 39 & $44 \cdot 3$ \\
\hline $0-0.4$ & 25 & 5 & $20 \cdot 0$ & $0-0.4$ & 113 & 38 & 33.6 & $0-0.35$ & 11 & 4 & 36.4 \\
\hline $0.4-0.8$ & 41 & 8 & 19.5 & $0.4-0.8$ & 80 & 32 & $40 \cdot 0$ & $0.35-0.7$ & 7 & 2 & 28.6 \\
\hline $0.8-1 \cdot 2$ & 46 & 6 & $13 \cdot 0$ & $0.8-1.2$ & 85 & 22 & $25 \cdot 9$ & $0.7-1.05$ & 18 & 7 & 38.9 \\
\hline $1 \cdot 2-1 \cdot 6$ & 20 & 6 & $30 \cdot 0$ & $1 \cdot 2-1 \cdot 6$ & 84 & 36 & $42 \cdot 9$ & $1.05-1.4$ & 10 & 5 & $50 \cdot 0$ \\
\hline $1 \cdot 6-2$ & 25 & 4 & $16 \cdot 0$ & $1 \cdot 6-2$ & 109 & 33 & $30 \cdot 3$ & $1.4-1.75$ & 11 & 5 & $45 \cdot 5$ \\
\hline $2-2.4$ & 18 & 4 & $22 \cdot 2$ & $2-2 \cdot 4$ & 58 & 24 & 41.4 & $1 \cdot 75-2 \cdot 1$ & 14 & 6 & 42.9 \\
\hline $2-2 \cdot 8$ & 10 & 2 & $20 \cdot 0$ & $2-2 \cdot 8$ & 53 & 20 & $37 \cdot 7$ & $2 \cdot 1-2 \cdot 45$ & 4 & 2 & $50 \cdot 0$ \\
\hline $2 \cdot 8-3 \cdot 2$ & 16 & 4 & $25 \cdot 0$ & $2 \cdot 8-3 \cdot 2$ & 39 & 14 & 35.9 & $>2.45$ & 63 & 29 & $46 \cdot 0$ \\
\hline$>3.2$ & 26 & 5 & $19 \cdot 2$ & $>3.2$ & 187 & 82 & 43.9 & & & & \\
\hline
\end{tabular}

GDM, gestational diabetes mellitus.

Table 4 Optimal ranges of gestational weight gain in the first trimester (GWG-F) for the avoidance of gestational diabetes mellitus in the study sample of pregnant women $(n 1910)$ from Southwest China, 2017 and normal-weight women, the optimal GWG-F range for overweight/obese women was lower. This result is consistent with previous findings ${ }^{(36,37)}$. Zhou et al., based on the Asian BMI classification, found that the mean GWG among Chinese women decreased as pre-pregnancy BMI increased. Zhang et al. observed that GWG-F was $0.92 \mathrm{~kg}$ among normal-weight women in China without any pregnancy complications ${ }^{(38)}$, which is similar to our findings. The IOM recommends the same range of weight gain in the first trimester regardless of pre-pregnancy BMI. This is the aspect of the IOM guidelines that is most different from our findings. Abrams et al. suggested that both the rate and the shape of the weight gain curve for relatively heavier women might differ from that of women with lower pre-pregnancy BMI ${ }^{(39)}$. Therefore, it was necessary to provide optimal GWG-F ranges according to the pre-pregnancy BMI. The optimal GWG-F ranges in our study were within IOM recommendations, while the upper limit for each pre-pregnancy BMI group was lower than that in the IOM recommendations.

Strengths and limitations should be considered when interpreting our findings. In terms of strengths, the present study shows the biological plausibility of a strong association between GWG-F and risk of GDM. Our study also adjusted for the effect of GWG-F on GDM when analysing the effect of RGWG-S on the risk of GDM. Further, in our investigation of the effects of GWG-F and RGWG-S on the risk of GDM and our exploration of the optimal GWG ranges for the avoidance of GDM, the pre-pregnancy BMI cut-off points were based on the China-specific BMI categories, which are more suitable for application in Chinese women. Another strength of the present study is that it established the first optimal GWG-F ranges stratified by China-specific BMI categories, which can be used for the avoidance of GDM and provide certain evidence for the establishment of GWG standards for Chinese women. Nevertheless, the present study also has some limitations. First, it used self-reported pre-pregnancy weight. Although high correlations have been found between self-reported 
and measured pre-pregnancy weight ${ }^{(12)}$, there may be potential misclassifications of pre-pregnancy BMI. There were also an insufficient number of women with high BMI to allow analyses of overweight and obese women separately, due to the demographics of overweight/obese women in Southwest China. In addition, our data are not from a nationally representative sample; the study used only a prospective cohort study in Chengdu, which is in the Southwest region of China, to eliminate the potential for selection bias. Due to the considerably high prevalence of GDM, the generalizability of our findings requires further investigation and more studies conducted in different areas or combined with another population are needed to verify the optimal ranges. Moreover, given the small sample sizes in the underweight and overweight/obese groups, the applicability of optimal GWG-F ranges for underweight and overweight/obese women is still limited. Further large studies are needed for verification as well.

\section{Conclusions}

Our study suggests that excessive GWG in the first trimester, rather than the second trimester, is associated with an increased risk of GDM regardless of pre-pregnancy BMI. The optimal GWG ranges in the first trimester for the avoidance of GDM are $0.8-1 \cdot 2,0.8-1.2$ and $0.35-0.7 \mathrm{~kg}$ for underweight, normal-weight and overweight/obese women, respectively. Identifying potential strategies for prevention of excessive GWG-F may have far-reaching benefits. Obstetricians should provide more pre-emptive nutrition guidance and lifestyle interventions in achieving adequate GWG-F. Further studies are needed to verify the generalizability of the ranges as well.

\section{Acknowledgements}

Acknowledgements: All participants are gratefully acknowledged. Financial support: All phases of this study were supported by a research grant from the Danone Nutrition Center Dietary Nutrition Research and Education Fund (grant number DIC2016-06). The funders had no additional role in the design, analysis or writing of this article. Conflict of interest: The authors reported no potential conflict of interest. Authorship: G.Z. designed the research; X.L. performed initial data analyses and wrote the manuscript; Y.Z., H.D. and J.Z. edited the manuscript; F.Z., Y.B. and C.C. performed data entry; R.Z., D.B. and X.P. provided critical input on the data analyses; G.Z. supervised the study. All authors critically reviewed and approved the final manuscript. Ethics of buman subject participation: This study was conducted according to the guidelines laid down in the Declaration of Helsinki and all procedures involving human subjects were approved by the Ethics Committee of Sichuan University.
Written informed consent was obtained from all the participants before they took part in the study.

\section{References}

1. American Diabetes Association (2004) Gestational diabetes mellitus. Diabetes Care 27, Suppl. 1, s88-s90.

2. Yogev Y, Xenakis EMJ \& Langer O (2004) The association between preeclampsia and the severity of gestational diabetes: the impact of glycemic control. Am J Obstet Gynecol 191, 1655-1660.

3. Metzger BE, Lowe LP, Dyer AR et al. (2008) Hyperglycemia and adverse pregnancy outcomes. $N$ Engl J Med 358, 1991-2002.

4. Marchetti D, Carrozzino D, Fraticelli F et al. (2017) Quality of life in women with gestational diabetes mellitus: a systematic review. J Diabetes Res 2017, 7058082.

5. Kim C, Newton KM \& Knopp RH (2002) Gestational diabetes and the incidence of type 2 diabetes: a systematic review. Diabetes Care 25, 1862-1868.

6. Feig DS, Corcoy R, Jensen DM et al. (2015) Diabetes in pregnancy outcomes: a systematic review and proposed codification of definitions. Diabetes Metab Res Rev 31, 680-690.

7. Zhang F, Dong L, Zhang CP et al. (2011) Increasing prevalence of gestational diabetes mellitus in Chinese women from 1999 to 2008. Diabet Med 28, 652-657.

8. Zhu W, Fan L, Yang H et al. (2013) Fasting plasma glucose at 24-28 weeks to screen for gestational diabetes mellitus: new evidence from China. Diabetes Care 36, 2038-2040.

9. Institute of Medicine \& National Research Council Committee to Reexamine IOM Pregnancy Weight Guidelines (2009) Weight Gain During Pregnancy: Reexamining the Guidelines. Washington, DC: National Academies Press.

10. Nohr EA, Vaeth M, Baker JL et al. (2008) Combined associations of prepregnancy body mass index and gestational weight gain with the outcome of pregnancy. Am J Clin Nutr 87, 1750-1759.

11. Morisset AS, Tchernof A, Dube MC et al. (2011) Weight gain measures in women with gestational diabetes mellitus. $J$ Womens Health 20, 375-380.

12. Hedderson MM, Gunderson EP \& Ferrara A (2010) Gestational weight gain and risk of gestational diabetes mellitus. Gynecol Obstet 115, 597-604.

13. Brunner S, Stecher L, Ziebarth S et al. (2015) Excessive gestational weight gain prior to glucose screening and the risk of gestational diabetes: a meta-analysis. Diabetologia 58, 2229-2237.

14. Liu Z, Ao D, Yang H et al. (2014) Gestational weight gain and risk of gestational diabetes mellitus among Chinese women. Chin Med J 127, 1255-1260.

15. Cho EH, Hur J \& Lee KJ (2015) Early gestational weight gain rate and adverse pregnancy outcomes in Korean women. PLoS One 10, e0140376.

16. Gibson KS, Waters TP \& Catalano PM (2012) Maternal weight gain in women who develop gestational diabetes mellitus. Gynecol Obstet 119, 560-565.

17. Institute of Medicine, Subcommittee on Nutritional Status During Pregnancy and Lactation (1990) Nutrition During Pregnancy: Part I, Weight Gain: Part II, Nutrient Supplements. Washington, DC: National Academies Press.

18. Headen I, Cohen AK, Mujahid M et al. (2017) The accuracy of self-reported pregnancy-related weight: a systematic review. Obes Rev 18, 350-369.

19. Yang HX, Sun WJ, Cu XM et al. (2011) Diagnosis Criteria for Gestational Diabetes Mellitus. Beijing: Ministry of Public Health.

20. Zhou B (2002) Predictive values of body mass index and waist circumference to risk factors of related diseases in 
Chinese adult population. Zhonghua Liu Xing Bing Xue Za Zhi 23, 5-10.

21. Ouyang F, Shen F, Jiang F et al. (2002) Risk factors in women with gestational diabetes mellitus. Zhonghua Yu Fang Yi Xue Za Zhi 36, 378-381.

22. Badon SE, Enquobahrie DA, Wartko PD et al. (2017) Healthy lifestyle during early pregnancy and risk of gestational diabetes mellitus. Am J Epidemiol 186, 326-333.

23. Torloni MR, Betran AP, Horta BL et al. (2009) Prepregnancy BMI and the risk of gestational diabetes: a systematic review of the literature with meta-analysis. Obes Rev 10, 194-203.

24. Gong LL, Liu H \& Liu LH (2016) Relationship between hypothyroidism and the incidence of gestational diabetes: a meta-analysis. Taiwan J Obstet Gynecol 55, 171-175.

25. Zhao WJ, Che QH \& Jia LH (2009) Case-control study on effect factors of gestational diabetes mellitus. Zhongguo Fu You Bao Jian 24, 3550-3552.

26. Dong B, Yu H, Wei Q et al. (2017) The effect of prepregnancy body mass index and excessive gestational weight gain on the risk of gestational diabetes in advanced maternal age. Oncotarget 8, 58364-58371.

27. Hantoushzadeh S, Sheikh M, Bosaghzadeh Z et al. (2016) The impact of gestational weight gain in different trimesters of pregnancy on glucose challenge test and gestational diabetes. Postgrad Med J 92, 520-524.

28. Zhong C, Li X, Chen R et al. (2017) Greater early and mid-pregnancy gestational weight gain are associated with increased risk of gestational diabetes mellitus: a prospective cohort study. Clin Nutr ESPEN 22, 48-53.

29. Carreno CA, Clifton RG, Hauth JC et al. (2012) Excessive early gestational weight gain and risk of gestational diabetes mellitus in nulliparous women. Gynecol Obstet 119, 1227-1233.
30. Saldana TM, Siega-Riz AM, Adair LS et al. (2006) The relationship between pregnancy weight gain and glucose tolerance status among black and white women in central North Carolina. Am J Obstet Gynecol 195, 1629-1635.

31. Herring SJ, Oken E, Rifas-Shiman SL et al. (2009) Weight gain in pregnancy and risk of maternal hyperglycemia. Am J Obstet Gynecol 201, 61.e1-61.e7.

32. Van Raaij JM, Peek ME, Vermaat-Miedema SH et al. (1988) New equations for estimating body fat mass in pregnancy from body density or total body water. Am J Clin Nutr 48, 24-29.

33. Macdonald SC, Bodnar LM, Himes KP et al. (2017) Patterns of gestational weight gain in early pregnancy and risk of gestational diabetes mellitus. Epidemiology 28, 419-427.

34. Bracero LA \& Byrne DW (1998) Optimal maternal weight gain during singleton pregnancy. Gynecol Obstet Invest 46, 9-16.

35. Juan DU, Qi XI \& Min LX (2011) The optimal range of gestational weight gain for singleton term nulliparous pregnancies in north region of China. Zhongguo Fu You Bao Jian 26, 2112-2114.

36. Zhou YB, Li HT, Ye RW et al. (2017) Gestational weight gain, cesarean delivery, and cesarean delivery on maternal request: a cohort analysis of Chinese nulliparous women. Ann Epidemiol 27, 96-102.e3.

37. Wong W, Tang NLS, Lau TK et al. (2000) A new recommendation for maternal weight gain in Chinese women. J Am Diet Assoc 100, 791-796.

38. Zhang CX, Huang WQ, Ming X et al. (2018) Weight gain during pregnancy and and its change: a prospective study. Wei Sheng Yan Jiu 47, 662-665.

39. Abrams B, Carmichael S \& Selvin S (1995) Factors associated with the pattern of maternal weight gain during pregnancy. Obstet Gynecol 86, 170-176. 